\title{
Paraquat Poisoning: A Retrospective Study of 55 Patients From a Tertiary Care Center in Southern India
}

\author{
R Ravichandran ${ }^{1}$, Deepak Amalnath², Kusa K Shaha ${ }^{3}$, BH Srinivas ${ }^{4}$
}

\begin{abstract}
Background: In India the data on paraquat (PQ) poisoning are limited to case reports and small case series. Hence, this study was carried out to understand the clinical features and outcomes of $\mathrm{PQ}$ poisoning. We also briefly report the relevant Indian studies on $\mathrm{PQ}$ poisoning. Materials and methods: This was a retrospective case record-based study of PQ poisoning victims admitted over a period of 5 years.

Results: Of the 55 patients included in this study, the in-hospital mortality rate was $72.7 \%$. Acute kidney injury was the most common manifestation. The use of cyclophosphamide did not affect the clinical outcome. Hemoperfusion (HP) was not done for any patient. Pulmonary edema and acute tubular necrosis were the most common histopathological findings.

Conclusion: In India, this is one of the most comprehensive studies of PQ toxicity. Hence, we hope that this information would be of use to clinicians who deal with $\mathrm{PQ}$ poisoning.

Keywords: Paraquat, Paraquat poisoning, Toxicology.

Indian Journal of Critical Care Medicine (2020): 10.5005/jp-journals-10071-23369
\end{abstract}

\section{INTRODUCTION}

Paraquat is a commonly used herbicide in India. ${ }^{1}$ Suicides due to paraquat $(\mathrm{PQ})$ are an important cause of morbidity and mortality, especially due to the absence of specific antidote. Metabolism of $\mathrm{PQ}$ generates free radicals that damage the cellular organelles and membranes, causing damage to many organs, especially the pulmonary alveolar epithelium. ${ }^{2}$

Consumption of $\mathrm{PQ}>40 \mathrm{mg} / \mathrm{kg}$ causes acute multiorgan failure with death within the first 2 days, while $<20 \mathrm{mg} / \mathrm{kg}$ of PQ causes mild symptoms and most survive. ${ }^{3}$

Paraquat of $20-40 \mathrm{mg} / \mathrm{kg}$ causes severe mucosal damage followed by multiorgan failure. The few, who survive, die within 2-4 weeks due to lung fibrosis. The pulmonary alveolar cells selectively accumulate polyamines that are required for cellular functions. Being structurally similar to polyamines, $P Q$ gets accumulated in these cells, causing selective delayed lung injury. 4

Although there is no specific antidote, most patients receive steroids/cyclophosphamide/antioxidants to reduce free radical damage.

Although PQ is commonly used in India, except for one large study, ${ }^{5}$ data on PQ poisoning are restricted to case reports and small case series. Hence, this study was undertaken to know the clinical features and outcome following PQ poisoning. We also briefly report the relevant Indian data on PQ poisoning.

\section{Materials and Methods}

This was a retrospective study of case records of patients with $P Q$ poisoning admitted to medicine wards over a period of 5 years (January 2014 to December 2018).

All consecutive patients of age more than 12 years were included in this study. Diagnosis was based on history of PQ intake and/or identifying the container with $\mathrm{PQ}$. Those with prior renal/ hepatic or lung diseases were not excluded.

Institute Scientific Committee and Ethics Committee approvals were obtained.

\begin{abstract}
1,2Department of Medicine, Jawaharlal Institute of Postgraduate Medical Education and Research, Puducherry, India

${ }^{3}$ Department of Forensic Medicine and Toxicology, Jawaharlal Institute of Postgraduate Medical Education and Research, Puducherry, India

${ }^{4}$ Department of Pathology, Jawaharlal Institute of Postgraduate Medical Education and Research, Puducherry, India

Corresponding Author: Deepak Amalnath, Department of Medicine, Jawaharlal Institute of Postgraduate Medical Education and Research, Puducherry, India, Phone: +91 9442533124, e-mail: deepakatgjipmer@ gmail.com
\end{abstract}

How to cite this article: Ravichandran R, Amalnath D, Shaha KK, Srinivas BH. Paraquat Poisoning: A Retrospective Study of 55 Patients from a Tertiary Care Center in Southern India. Indian J Crit Care Med 2020;24(3):155-159.

Source of support: Nil

Conflict of interest: None

A pro forma was used to collect data regarding the demographics, symptoms, complications, outcomes, treatment with immunosuppression, laboratory, and radiological findings. Amount of PQ ingested was classified as mild $(<10 \mathrm{~mL})$, moderate $(10-20 \mathrm{~mL})$, and severe $(>20 \mathrm{~mL}){ }^{4}$

Lung involvement was diagnosed based on hypoxemia and infiltrates on chest X-ray. Kidney injury was diagnosed by raising creatinine/oliguria/need for dialysis. The use of steroids and cyclophosphamide was decided on case-to-case basis by the treating units, and no specific protocol was used for the same.

Mortality included patients who were taken against medical advice, when they deteriorated due to refractory hypotension/ multiorgan failure. Postmortem records were analyzed to know the organs involved and their histological features.

\section{Statistical Analysis}

Descriptive statistics was used to denote baseline characteristics. Chi-square test was used to study the association between modality of treatment and mortality.

o The Author(s). 2020 Open Access This article is distributed under the terms of the Creative Commons Attribution 4.0 International License (https://creativecommons. org/licenses/by-nc/4.0/), which permits unrestricted use, distribution, and non-commercial reproduction in any medium, provided you give appropriate credit to the original author(s) and the source, provide a link to the Creative Commons license, and indicate if changes were made. The Creative Commons Public Domain Dedication waiver (http://creativecommons.org/publicdomain/zero/1.0/) applies to the data made available in this article, unless otherwise stated. 


\section{Results}

A total of 55 patients were included in this study (Table 1$)$.

Most of them were males (65.4\%) and the median age was 28 years. Many patients (54.5\%) presented by 6-12 hours after ingestion of $\mathrm{PQ}$ and $54.5 \%$ had consumed moderate amount of PQ. Hypotension was documented in $32.7 \%$. Lung injury was documented in $61.8 \%$ and $18.1 \%$ needed mechanical ventilation. Of the 35 who had undergone chest X-ray, 10 had lung infiltrates. Computed tomography chest was done for two patients, which was normal. Paraquat tongue was documented in six patients.

Acute kidney injury was documented in $81.8 \%$, and $56.3 \%$ had undergone dialysis.

Details of liver function tests were available for 45 patients, of whom $33.3 \%$ had bilirubin $>2 \mathrm{mg} / \mathrm{dL}$. Elevation of (more than two times the upper normal limit) aspartate (AST), alanine aminotransferase (ALT) and alkaline phosphatase (ALP) was reported in $46.4 \%, 46.4 \%$, and $24.4 \%$, respectively. Acute hepatic failure was not noted in anyone.

Thirty-nine patients (70.9\%) had received steroids (pulse methyl prednisolone: 29 , hydrocortisone: 6 , prednisolone: 3 , and dexamethasone: 1) and cyclophosphamide (750 mg intravenously for 1-2 days, along with steroids) in $30.9 \%$. The use of steroids and cyclophosphamide did not lead to a decrease in mortality rate (suppl. Tables S1 and S2).

Table 1: Clinical features and laboratory findings

\begin{tabular}{lc}
\hline Baseline characteristics & \\
Male & $36(65.5 \%)$ \\
Female & $19(34.5 \%)$ \\
Age & \\
$\quad<20$ & $7(12.7 \%)$ \\
$20-40$ & $38(69.1 \%)$ \\
$41-84$ & $10(18.2 \%)$ \\
Amount consumed & \\
Mild & $13(23.6 \%)$ \\
Moderate & $30(54.5 \%)$ \\
$\quad$ Severe & $12(21.9 \%)$ \\
Time to admission & \\
$\quad<6$ hours & $12(21.8 \%)$ \\
$6-24$ hours & $30(54.5 \%)$ \\
$>24$ hours & $13(23.7 \%)$ \\
Hypotension & $18(32.7 \%)$ \\
Lung injury & $34(61.8 \%)$ \\
Acute kidney injury & $45(81.5 \%)$ \\
Dialysis & $31(56.3 \%)$ \\
Liver function tests $(n=45)$ & \\
Total bilirubin $>2 \mathrm{mg} / \mathrm{dL}$ & $15(33.3 \%)$ \\
AST $>2$ UNL & \\
ALT $>2$ UNL & $21(46.6 \%)$ \\
ALP $>2$ UNL & $14(31.1 \%)$ \\
Serum albumin $<3.5 \mathrm{~g} / \mathrm{dL}$ & $21(46.6 \%)$ \\
Serum creatinine $>2 \mathrm{mg} / \mathrm{dL}$ & $19(42.2 \%)$ \\
\hline & $11(24.4 \%)$ \\
\hline & $4(8.8 \%)$ \\
\hline & $6(13.3 \%)$ \\
\hline & $39(70.9 \%)$ \\
\hline
\end{tabular}

Outcome: During hospitalization, 35 died and 5 were discharged against medical advice (mortality rate of $72.7 \%$ ). All patients died within 3 days of PQ consumption. Most common cause of death was acute lung and kidney injury. Those who were discharged were later contacted by phone. Increased quantity of $\mathrm{PQ}$ consumed had higher mortality, while the time of presentation did not affect the mortality rate (suppl. Table S3 and S4). Presence of lung injury had higher mortality rate, while kidney injury did not have any correlation with mortality rate (suppl. Tables S5 and S6).

Of the 15 survivors, 7 could not be contacted, 5 were alive, and 3 died later elsewhere (within 2 months, likely due to delayed pulmonary fibrosis).

Autopsy was done on 30 patients (Table 2). Lungs, liver, and kidneys were sent for histopathological analysis. Lung was involved in all cases, with pulmonary edema and hemorrhage being the most common findings. Chronic venous congestion of the liver and renal acute tubular necrosis were the other important findings (Fig. 1). No characteristic histological findings were observed that suggested $\mathrm{PQ}$ poisoning.

\section{Discussion}

In this retrospective study of 55 patients, most were males in the age-group of 20-40 years. Acute lung and renal involvement led to high mortality rate of $72.5 \%$, with at least three more dying after discharge due to delayed lung fibrosis. The use of immunosuppression did not affect the outcome.

We tried to compare our data with other studies from India. Except for one study by Rao et al., ${ }^{5}$ most of the reported data are limited to small series of less than 10 patients or case reports (Table 3). Of the 101 patients studied by Rao et al., the mortality rate was $61.4 \%$ which is less than that in our study. This may be because patients who left against medical advice were excluded. Also, delayed mortality due to lung fibrosis has not been reported in any of the Indian studies, while 3 of the 15 of our patients died due to lung fibrosis. This suggests that the so-called survivors should be kept on close follow-up and the overall mortality rates could be higher than reported.

\begin{tabular}{lc} 
Table 2: Autopsy findings & \\
\hline Lung $(n=30)$ & 12 \\
Congestion & 19 \\
Hemorrhage & 3 \\
Pneumonia & 2 \\
$\quad$ Normal & \\
Liver ( $n=25)$ & 5 \\
Hepatitis & 3 \\
Chronic venous congestion & 3 \\
Cholestasis & 3 \\
Steatosis & 14 \\
Normal & \\
Kidney ( $n=24)$ & 5 \\
Glomerular changes & 17 \\
Acute tubular necrosis & 2 \\
Hemorrhage & 5 \\
Normal & \\
Heart ( $n=7)$ & 1 \\
Hemorrhage & \\
\hline Note: some patients had more than one findings in the involved organs
\end{tabular}




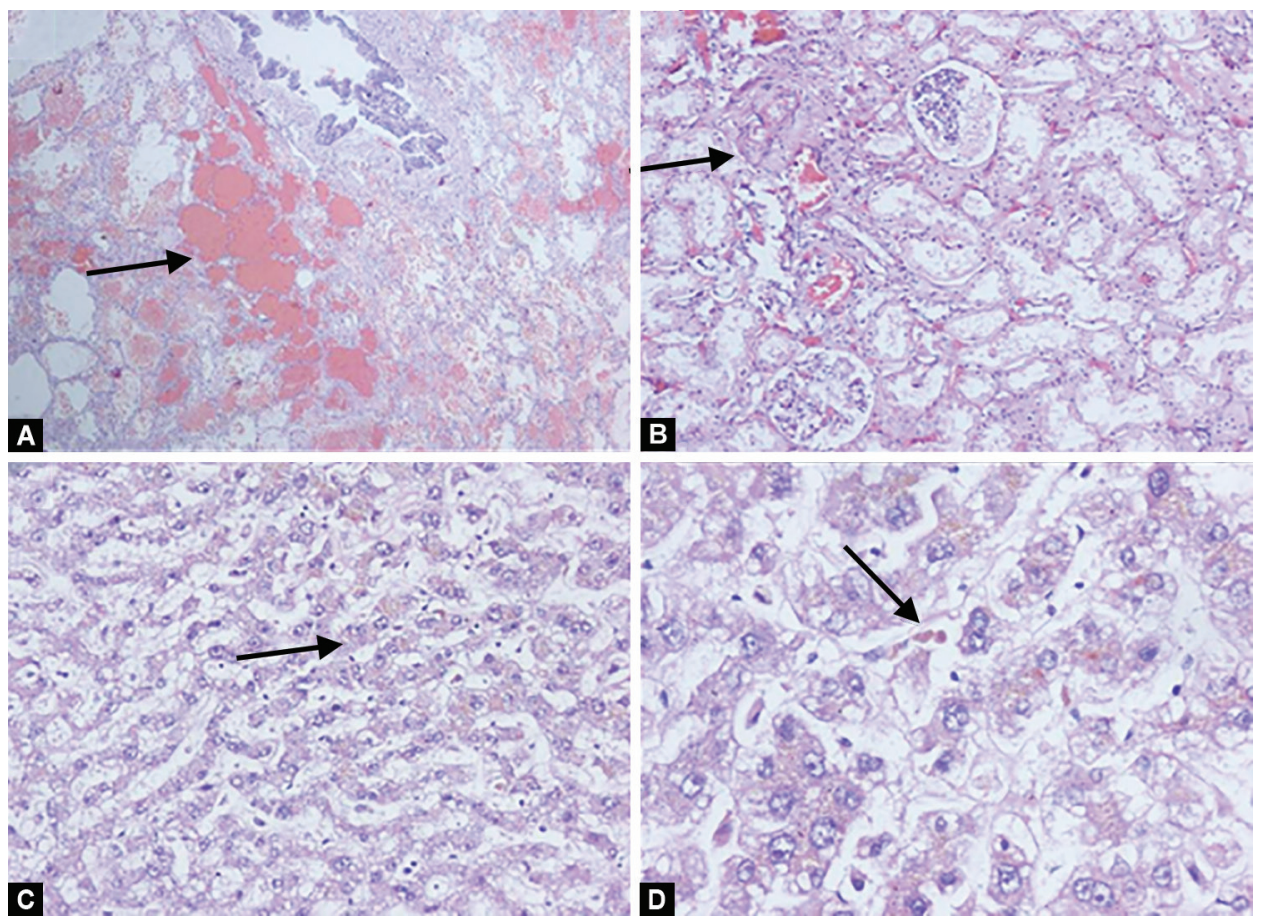

Figs 1 A to D: Histopathological sections from (A) Lung shows congestion and alveolar hemorrhage (H\&E $\times 40)$; (B) Kidney shows evidence of congestion in glomerular and interstitial capillaries with mild acute tubular necrosis (H\&E $\times 100) ;(C$ and D) Liver shows intracytoplasmic and intracanalicular cholestasis (arrow) and microvesicular steatosis (arrow head) (H\&E $\times 100)$

Table 3: Indian studies on paraquat poisoning

\begin{tabular}{|c|c|c|c|c|c|c|c|c|}
\hline Study duration & $\begin{array}{l}\text { Number of } \\
\text { patients }\end{array}$ & Mortality rate (\%) & Steroids & Cyclo (\%) & Vit $C / E$ & $N A C$ & $H P(\%)$ & Autopsy \\
\hline Rao et al..$^{5}$ (4 years) & 101 & 61.4 & $\mathrm{~N}$ & $\mathrm{~N}$ & $\mathrm{Y}$ & $\mathrm{N}$ & $\mathrm{Y}(62.4)$ & $\mathrm{N}$ \\
\hline This study (5 years) & 55 & 72.7 & $\mathrm{Y}$ & $\mathrm{Y}$ & $\mathrm{N}$ & $\mathrm{N}$ & $\mathrm{N}$ & Y \\
\hline Sandhu et al. ${ }^{14}$ (5 years) & 17 & 58.2 & Y & $\mathrm{N}$ & Y & $\mathrm{N}$ & $\mathrm{N}$ & $\mathrm{N}$ \\
\hline Narendra et al. ${ }^{7}$ ( 2 years) & 8 & 75 & Y & Y & $\mathrm{Y}$ & $\mathrm{Y}$ & $\mathrm{N}$ & $\mathrm{N}$ \\
\hline $\operatorname{Pavan}^{15}$ ( 2 years) & 6 & 66.7 & Y & $\mathrm{N}$ & $\mathrm{N}$ & $\mathrm{N}$ & $\mathrm{N}$ & $\mathrm{N}$ \\
\hline Banday et al. ${ }^{16}$ (1 year) & 8 & 75 & Y & $\mathrm{N}$ & $\mathrm{N}$ & $\mathrm{N}$ & $\mathrm{N}$ & $\mathrm{N}$ \\
\hline Jagadeesan et al. ${ }^{17}$ (3 years) & 10 & 100 & Not mentioned & $Y(50)$ & & & & \\
\hline Prasad et al. ${ }^{12}$ ( 2 years) & 6 & 83.3 & Not mentioned & $\mathrm{N}$ & $\mathrm{Y}$ & & & \\
\hline Kanchan et al. ${ }^{11}$ ( 2 years) & 14 & $\begin{array}{l}\text { Autopsy study of } 14 \text { patients. } \\
\text { Treatment details not available }\end{array}$ & & & & & & \\
\hline
\end{tabular}

Cyclo, cyclophosphamide; NAC, $N$ acetyl cysteine; HP, hemoperfusion; $\mathrm{Y}$, yes; N, no

\section{Efficacy of Cyclophosphamide and HP}

Although small series reported beneficial effects of cyclophosphamide, a recent randomized controlled trial showed no benefit over placebo. ${ }^{6}$ Of the eight patients reported by Narendra et al., ${ }^{7}$ all died despite receiving cyclophosphamide. In our study, $27.2 \%$ received cyclophosphamide, but it did not reduce mortality.

Evidence for possible beneficial role of $\mathrm{HP}$ in $\mathrm{PQ}$ poisoning has been restricted to case series and retrospective studies. Rao et al. ${ }^{5}$ reported that early HP may be of benefit. However, $\mathrm{PQ}$ is rapidly excreted by the kidneys, $80-90 \%$ by 6 hours and $100 \%$ by 24 hours. ${ }^{3}$ Hence, HP may not offer added benefit in those patients who have preserved renal function, at least in the first 24 hours. In our study, hemodialysis was done in $50.4 \%$ but HP was not done for anyone. Although Li et al. ${ }^{8}$ showed a moderate benefit of combining HP with continuous venovenous hemofiltration, another study by Koo et al. ${ }^{9}$ did not find any advantage of this therapy. Paraquat could still be detected in the viscera of those who died despite receiving HP and immunosuppression, suggesting that these measures may not be of use in $\mathrm{PQ}$ poisoning. ${ }^{10}$

\section{Autopsy Findings}

Congestion and edema of the viscera (especially lungs) and brain have been reported by Kanchan et al. ${ }^{11}$ (14 patients) and by Prasad et al. ${ }^{12}$ (5 patients). In our study, congestion and hemorrhage were the most common findings in the lung. Pulmonary fibrosis was not documented, since all autopsy patients had died during the acute phase of PQ poisoning. Kidney involvement in the form of acute tubular necrosis (ATN) is expected, since PQ has rapid renal elimination. In only two patients, PQ could be detected in the viscera. This may because of the rapid elimination of $\mathrm{PQ}$. 


\section{LIMITATIONS}

Since this is a retrospective study, laboratory data were not available for some patients. Biochemical confirmation at bedside was not done. This study was not powered to assess the efficacy of cyclophosphamide in $\mathrm{PQ}$ poisoning. Follow-up data were missing for seven patients.

\section{Conclusion}

Our study showed a very high in-hospital mortality rate of $72.7 \%$. The amount of PQ consumed and the presence of lung injury had correlation with mortality rate, while the use of steroids/ cyclophosphamide did not lead to reduced mortality rate.

In the absence of effective antidote and specific treatment, $P Q$ continues to cause very high mortality rates. In many countries (Sri Lanka, South Korea), PQ has been banned, ${ }^{2,13}$ which were followed by reduction in pesticide-associated mortality rates. We hope that this study will help clinicians in the management of $\mathrm{PQ}$ poisoning.

\section{References}

1. Kumar D, Conditions of Paraquat use in India. Available from: http:// www.iuf.org/w/sites/default/files/Joint $\% 20$ paraquat $\% 20$ study $\% 20$ India_0.pdf. [Accessed 1st August 2019].

2. Gawarammana IB, Buckley NA. Medical management of paraquat ingestion. Br J Clin Pharmacol 2011;72(5):745-757. DOI: 10.1111/j.13652125.2011.04026.x.

3. Dinis-Oliveira RJ, Duarte JA, Sanchez-Navarro A, Remiao F, Bastos $\mathrm{ML}$, Carvalho F. Paraquat poisonings: mechanisms of lung toxicity, clinical features, and treatment. Crit Rev Toxicol 2008;38(1):13-71. DOI: 10.1080/10408440701669959.

4. Hoet PH, Nemery B. Polyamines in the lung: polyamine uptake and polyamine-linked pathological or toxicological conditions. Am J Physiol Lung Cell Mol Physiol 2000;278(3):L417-L433. DOI: 10.1152/ ajplung.2000.278.3.L417.

5. Rao R, Bhat R, Pathadka S, Chenji SK, Dsouza S. Golden hours in severe paraquat poisoning-the role of early haemoperfusion therapy. J Clin Diag Res 2017;11(2):OC06. DOI: 10.7860/JCDR/2017/24764.9166.
6. Gawarammana I, Buckley NA, Mohamed F, Naser K, Jeganathan $\mathrm{K}$, Ariyananada PL, et al. High-dose immunosuppression to prevent death after paraquat self-poisoning - a randomized controlled trial. Clin Toxicol (Phila) 2018;56(7):633-639. DOI: 10.1080/15563650.2017.1394465.

7. Narendra SS,Dr, Vinaykumar. S. Paraquat poisoning: a case series in South India. Int J Sci Res 2015;4(1):561-564.

8. Li C, Hu D, Xue W, Li X, Wang Z, Ai Z, et al. Treatment outcome of combined continuous venovenous hemofiltration and hemoperfusion in acute paraquat poisoning: a Prospective controlled trial. Crit Care Med 2018;46(1):100-107. DOI: 10.1097/ CCM.0000000000002826.

9. Koo JR, Kim JC, Yoon JW, Kim GH, Jeon RW, Kim HJ, et al. Failure of continuous venovenous hemofiltration to prevent death in paraquat poisoning. Am J Kidney Dis 2002;39(1):55-59. DOI: 10.1053/ ajkd.2002.29880.

10. Dinis-Oliveira RJ, de Pinho PG, Santos L, Teixeira H, Magalhães T, Santos A, et al. Postmortem analyses unveil the poor efficacy of decontamination, anti-inflammatory and immunosuppressive therapies in paraquat human intoxications. PLoS ONE 2009;4(9):e7149. DOI: 10.1371/journal.pone.0007149.

11. Kanchan T, Bakkannavar SM, Acharya PR. Paraquat poisoning: Analysis of an uncommon cause of fatal foisoning from Manipal, South India. Toxicol Int 2015;22(1):30-34. DOI: 10.4103/0971-6580.172253.

12. Prasad DRMM, Chennabasappa A. Outcome of paraquat Poisoned patients Treated with a commonly used therapeutic flowchart: a case series. Asia Pac J Med Toxicol 2015;4:139-142.

13. Cha ES, Chang SS, Gunnell D, Eddleston M, Khang YH, Lee WJ. Impact of paraquat regulation on suicide in South Korea. Int J Epidemiol 2016;45(2):470-479. DOI: 10.1093/ije/dyv304.

14. Sandhu JS, Dhiman A, Mahajan R, Sandhu P. Outcome of paraquat poisoning - a five year study. Indian J Nephrol 2003;13:64-68.

15. Pavan M. Acute kidney injury following paraquat poisoning in india. Iran J Kidney Dis 2013;7(1):64-66.

16. Banday TH, Bhat SB, Bhat SB. Manifestation, complications and clinical outcome in paraquat poison: a hospital based study in a rural area of Karnataka. J Environ Occup Sci 2014;3(1):21-24. DOI: 10.5455/ jeos.20140127031530.

17. Jagadeesan $\mathrm{M}$, Nithyananthan $\mathrm{P}$, Banupriya $\mathrm{M}$, Mahendrakumar $\mathrm{K}$, Prasanna Karthik S, Kannan R. A study on clinical profile of paraquat poisoning in a tertiary care hospital. Int J Adv Med 2017;4(4): 1088-1091. DOI: 10.18203/2349-3933.ijam20173237. 
Paraquat Poisoning: A Retrospective Study of 55 Patients From a Tertiary Care Center in Southern India

Table S1: Comparing steroids with mortality

\begin{tabular}{llrrr}
\hline & & \multicolumn{3}{c}{ Mortality } \\
\cline { 3 - 4 } & & Yes & No & Total \\
\hline Steroids & Yes & 26 & 13 & 39 \\
& No & 14 & 2 & 16 \\
Total & & 40 & 15 & \\
\hline$P=0.11$ & & & &
\end{tabular}

$p=0.11$

Table S2: Comparing cyclophosphamide with mortality

\begin{tabular}{llrrr}
\hline & & \multicolumn{3}{c}{ Mortality } \\
\cline { 3 - 4 } & & Yes & No & Total \\
\hline Cyclophosphamide & Yes & 11 & 6 & 17 \\
& No & 29 & 9 & 38 \\
Total & & 40 & 15 & \\
\hline
\end{tabular}

$p=0.37$

Table S3: Comparing the time of presentation with mortality

\begin{tabular}{lrrr}
\hline & \multicolumn{3}{c}{ Mortality } \\
\cline { 2 - 3 } & Yes & No & Total \\
\hline$<6$ Hours & 7 & 5 & 12 \\
6-24 Hours & 24 & 6 & 30 \\
$>24$ Hours & 9 & 4 & 13 \\
Total & 40 & 15 & \\
\hline$P=0.34$ & & & \\
\hline
\end{tabular}

Table S4: Comparing the amount of paraquat consumed with mortality

\begin{tabular}{lcrl}
\hline & \multicolumn{3}{c}{ Mortality } \\
\cline { 2 - 3 } & Yes & No & Total \\
\hline$<50 \mathrm{~mL}$ & 4 & 9 & 13 \\
$50-100 \mathrm{~mL}$ & 25 & 5 & 30 \\
$>100 \mathrm{~mL}$ & 11 & 1 & 12 \\
Total & 40 & 15 & \\
\hline
\end{tabular}

$p=0.001$

Table S5: Comparing lung injury with mortality

\begin{tabular}{lllll}
\hline & & \multicolumn{3}{c}{ Mortality } \\
\cline { 3 - 4 } & & Yes & No & Total \\
\hline Lung injury & Yes & 30 & 4 & 34 \\
& No & 10 & 11 & 21 \\
Total & & 40 & 15 & \\
\hline$p=0.001$ & & & &
\end{tabular}

Table S6: Comparing kidney injury with mortality

\begin{tabular}{lllll}
\hline & & \multicolumn{2}{l}{ Mortality } & \\
\cline { 3 - 5 } & & Yes & No & Total \\
\hline Kidney injury & Yes & 34 & 11 & 45 \\
& No & 6 & 4 & 10 \\
Total & & 40 & 15 & \\
\hline$p=0.31$ & & &
\end{tabular}

